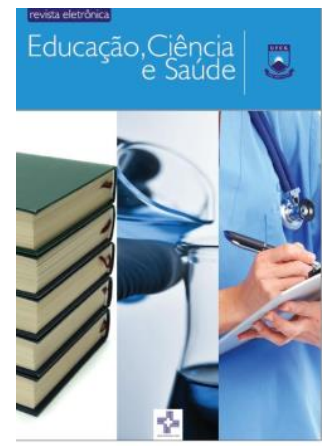

EDUCAÇÃO CIÊNCIA E SAÚDE

http://dx.doi.org/10.20438/ecs.v6i1.203

\title{
CUIDADOS DE ENFERMAGEM NOS CASOS DE INTOXICAÇÕES EXÓGENAS: REVISÃO INTEGRATIVA
}

\author{
Lília Costa Nascimento ${ }^{1}$, Adilma da Cunha Cavalcanti ${ }^{1}$, Miriam Maria Mota \\ Silva1 ${ }^{1}$, Darli Maria de Souza ${ }^{1}$, Adriana Montenegro de Albuquerque ${ }^{2}$ \\ ${ }^{1}$ Graduadas em Enfermagem pela Universidade Federal de Campina Grande, Cuité-PB, Brasil. \\ 2 Prof ${ }^{a}$ Unidade Acadêmica de Saúde, Universidade Federal de Campina Grande, Cuité, PB, \\ Brasil. \\ E-mail para correspondência: liliac323@gmail.com
}

\begin{abstract}
Resumo
As intoxicações exógenas são definidas como um conjunto de efeitos nocivos, que causam desequilíbrio orgânico, ocasionado por substâncias químicas, seja por via de inalação, digestiva, cutânea entre outras. Objetivou-se identificar as publicões científicas sobre os cuidados de enfermagem diante das intoxicações exógenas no Brasil. Trata-se de uma revisão integrativa, realizada nas bases de dados indexadoras: LILACS, SCIELO, BDENF, utilizando os descritores "Intoxicação/Envenenamento", "Cuidados de Enfermagem" e "Prevenção de acidentes", no período de janeiro a março de 2019, contabilizando 12 artigos considerando os anos de publicações entre 2013 a 2019. A intoxicação exógena pode ser apresentada de várias formas, sejam, por agentes ambientes, medicamentos, alimentos ou até mesmo a água, apresentando um efeito nocivo. Pode-se classificá-la em aguda ou crônica, que causam manifestações clínicas significantes e até fatais. Considera-se o papel da enfermagem como primordial no contexto do atendimento multiprofissional ao paciente com diagnóstico de intoxicação exógenas por diversas causas. Ressalta-se importância na articulação da assitência de enfermagem na promoção, prevenção e tratamento para esse agravo. Salienta-se a importância do enfermeiro está preparado cientificamente para cuidar de paciente acometidos com intoxicação exógena, visando não apenas o estado físico, mas o social e psicologico.
\end{abstract}

Palavras-chave: Intoxicação, Envenenamento, Cuidados de Enfermagem, Prevenção de Acidentes.

\begin{abstract}
Exogenous intoxications are defined as a set of harmful effects, which cause organic imbalance, caused by chemical substances, either by inhalation, digestive, cutaneous, among others. The objective was to identify the scientific publications on nursing care in the face of exogenous intoxications in Brazil. It is an integrative review, carried out in the index databases: LILACS, SCIELO, BDENF, using the descriptors "Poisoning / Intoxication", "Nursing Care" and "Accident Prevention", from January to March, 2019 , accounting for 12 articles considering the years of publication between 2013 to 2019. Exogenous intoxication can be presented in various ways, whether by environment agents, medicines, food or even water,
\end{abstract}


presenting a harmful effect. It can be classified as acute or chronic, which causes significant and even fatal clinical manifestations. The role of nursing is considered as paramount in the context of multiprofessional care to the patient diagnosed with exogenous intoxication due to several causes. Emphasis is placed on the articulation of nursing care in the promotion, prevention and treatment of this condition. It is emphasized the importance of the nurse is prepared scientifically to care for patients affected with exogenous intoxication, aiming not only the physical, but the social and psychological state.

Keywords: Intoxication, Poisoning, Nursing Care, Accident Prevention.

\section{Introdução}

Intoxicação exógena é o termo utilizado para designar uma reação do organismo perante a exposição frequente e excessiva a substâncias químicas, onde o efeito nocivo ocorre quando uma substância tóxica é ingerida ou adentram em contato com pele, olhos ou mucosas acarretando sinais, sintomas, e comprometimento órgãos e tecidos, podendo levar a morte (MELO et al., 2015).

O aumento progressivo é desordenado no consumo de substâncias químicas não acompanhadas de cuidados precisos causam sérios problemas com um alto risco a saúde. Frente a isto, no Brasil destaca-se número expressivo de pessoas intoxicadas com registros de casos de intoxicação, sejam pela ingestão de alimentos contaminados, medicamentos, uso de agrotóxicos, produtos de limpeza doméstica, e outras substâncias químicas (SANTOS; LEGAY; LOVISI, 2013).

As intoxicações podem ser consideradas agudas, quando ocorre após exposição única ou repetida ao agente químico no período de até 24 horas, sendo utilizada a inativação ou remoção do tóxico como tratamento de emergência (SANTOS; NETO; CUNHA, 2015).

Nos casos de intoxicação crônica ocorrem repetidas exposições, que normalmente duram longos períodos de tempo, ocasionando graves problemas neurológicos, imunológicos, endocrinológicos, renais, malformações congênitas, hematológicos, dermatológicos, hepáticos e tumores (BRASIL, 2014). As intoxicações exógenas são ocorrências frequentes que indicam uma procura dos setores de urgência e emergência em todo o mundo.

Os acidentes com intoxicações são consideradas um fenômeno emergente, devido o desenvolvimento industrial das áreas de química e farmácia, à facilidade de acesso a medicamentos e substâncias tóxicas, ao largo emprego de praguicidas nas áreas rurais e urbanas, à prescrição médica abusiva de psicotrópicos e à falta de 
cuidado adequado no manuseio de determinadas substâncias tóxicas (TAVARES; OLIVEIRA, 2012).

Segundo Santos, Legay e Lovisi (2013) os homens totalizam um número maior do total em comparação com as mulheres que comete tentativas de suicídio com substâncias tóxicas, sendo essa população mais prejudicada devido à maior incidência de exposição.

A equipe de enfermagem tem um papel relevante diante das intoxicações exógenas, através de ações de assistência a esses pacientes, por meio da Sistematização de Assistência de Enfermagem (SAE), voltada para o tipo específico de intoxicação, prevenindo possíveis complicações, e alterações orgânicas ocasionadas por agente químico. É necessária uma atuação multiprofissional em todas as etapas da assistência (MELO et al., 2015). A SAE é uma metodologia que vem cada vez mais conferir uma prática assistencial de qualidade e segura.

Diante disso este estudo tem como objetivo identificar e analisar as publicações científicas sobre a assistência de enfermagem diante das intoxicações exógenas no Brasil.

\section{METODOLOGIA}

Trata-se de uma revisão integrativa, construída a partir de fontes secundárias, através de um levantamento de materiais bibliográficos no meio eletrônico. Este estudo vem sendo utilizado como um meio metodológico, e também como um recurso sistemático que tem por objetivo reunir e sintetizar resultados de pesquisas enfocando um tema especifico, ou seja, determina o conhecimento atual sobre uma determinada temática especifica, tendo como características identificar, analisar e sintetizar os resultados encontrados (SOARES et al., 2014).

Segundo Soares et al. (2014), a revisão integrativa permite a síntese de um determinado assunto, sendo estruturada em seis etapas: 1a identificação do tema e seleção da hipótese ou questão de pesquisa para a elaboração da revisão integrativa, 2a formular e estabelecer critérios para inclusão e exclusão de estudos, amostragem e busca na literatura, $3^{\mathfrak{a}}$ definição das informações a serem extraídas dos estudos selecionados e categorização dos estudos, 4⿳亠丷a avaliação dos estudos incluídos na revisão integrativa, $5^{\mathbf{a}}$ interpretação dos resultados e $6^{\mathbf{a}}$ apresentação da revisão e síntese do conhecimento. 
$\mathrm{Na} 1^{\text {a }}$ etapa iniciou-se pela identificação do tema e problema de estudo: $\mathrm{A}$ questão norteadora do estudo foi: o que tem sido publicado nas bases de dados nacionais acerca das Intoxicações exógenas enfatizando os cuidados de enferrmagem a vítimas de envenenamento?

A 2a etapa: houve o estabelecimento de critérios de inclusão dos materiais que fizeram parte da revisão e busca da literatura nas bases de dados. Para compor o corpus da pesquisa buscaram-se artigos indexados online nas bases de dados: SciELO (Scientific eletronic Library Online), BDENF (Base de Dados de Enfermagem), LILACS (Literatura Latino Americana e do Caribe em Ciências da Saúde) as duas últimas incluídas na BVS (Biblioteca Virtual de Saúde). Para tanto, utilizou-se os descritores: "Intoxicação/Envenenamento", "Cuidados de Enfermagem" e "Prevenção de acidentes". Está pesquisa foi realizada entre os meses de janeiro a março de 2019.

Para a seleção da amostra, foram utilizados os seguintes critérios de inclusão: artigos com texto completo, na língua vernácula, publicados a partir do ano de 2013 até 2019, que de fato se relacionassem com o tema da presente revisão e estivessem disponíveis na internet e fossem gratuitos. Foram excluídos os artigos cujo texto completo não estivesse disponível na modalidade free, estudos secundários, carta ao leitor, teses, dissertações, artigos em outros idiomas

A pesquisa foi realizada de forma independente, por meio do cruzamento nas bases selecionadas. Desta forma, foram encontrados 2.086 artigos indexados nas bases de dados consultadas, sendo: 1.451 na base SCIELO a partir do cruzamento dos DECS (Descritores em Ciência da Saúde), "Envenenamento Or Cuidados de Enfermagem Or Prevenção de acidentes", 20 na base LILACS a partir do cruzamento dos DECS: "Envenenamento And Cuidados de Enfermagem"e 15 na BDENF com os DECS “"Envenenamento And Cuidados de Enfermagem”, após filtragem, análise criteriosa dos artigos e critérios de exclusão, foram selecionados 12 publicações, que se adequaram a questão norteadora e são objeto desta pesquisa de revisão integrativa. Permaneceu na amostra final após esse processo 8 artigos da base de dados SCIELO, 2 da LILACS e 2 da BDENF.

A seguir, o quadro 1 está representado pela seleção dos artigos pesquisados, eccluídos e selecionados por bases de dados. 
Quadro 1. Distribuição de artigos selecionados para revisão, segundo as bases de dados. Cuité (PB), 2019.

\begin{tabular}{|c|c|c|c|}
\hline \multirow{2}{*}{ ARTIGOS } & \multicolumn{3}{|c|}{ BASE DE DADOS } \\
\cline { 2 - 4 } & SCIELO & LILACS & BDENF \\
\hline Pesquisados & 1.451 & 4 & 4 \\
\hline Excluídos & 1.443 & 2 & 2 \\
\hline Selecionados & 8 & 2 & 2 \\
\hline Total & & 12 & \\
\hline
\end{tabular}

Fonte: Elaboração Própria

$\mathrm{Na} 3^{\underline{a}}$ etapa buscou-se ler e extrair as informações sobre a intoxicação exógena, assistência de enfermagem, fatores de risco, incidência e tratamento, para posterior discussão.

$\mathrm{Na} 4^{\underline{a}}$ etapa ou avaliação dos estudos incluídos na revisão, após a extração dos dados na terceira etapa, os mesmos foram categorizados e apresentados na discussão, para melhor possibilitar o entendimento do leitor, e melhor mostrar os achados.

$\mathrm{Na}$ interpretação dos resultados e $5^{\mathrm{a}}$ etapa, após a leitura foi feito a análise sobre a realidade do contexto atual da temática, avaliou-se recomendações, sugestões para pesquisas futuras, como estabelecido por (SOARES et al., 2014).

Na sexta e última etapa, conforme Souza, Silva e Carvalho (2010), consistiu na síntese e apresentação do conteúdo, trazendo resumo, detalhe sobre os achados, apresentação do conteúdo pesquisado. 


\section{RESULTADOS}

Foram analisados 12 artigos que respondiam aos critérios de inclusão previamente estabelecidos. Sendo que estas publicaçãoes apresentaram as respostas mais precisas para o objetivo da pesquisa, que estão descritas no quadro 2, na sequência dos autores, título, objetivo e ano de publicação.

Quadro 2: Distribuição dos artigos selecionados apresentado autor, título, objetivo, ano. Cuité (PB), 2019.

\begin{tabular}{|c|c|c|c|}
\hline $\mathbf{N r}$ & Autores/Ano & Título & Objetivo \\
\hline 1 & $\begin{array}{l}\text { TAVARES et al., } \\
2013\end{array}$ & $\begin{array}{l}\text { Fatores associados à } \\
\text { intoxicação infantil }\end{array}$ & $\begin{array}{l}\text { Analisar os fatores associados à } \\
\text { intoxicação em crianças, a partir } \\
\text { de casos registrados no centro } \\
\text { de controle de intoxicações do } \\
\text { Hospital Universitário de } \\
\text { Maringá. }\end{array}$ \\
\hline 2 & REIS et al., 2013 & $\begin{array}{l}\text { Saúde do homem: } \\
\text { Internações } \\
\text { hospitalares por } \\
\text { intoxicação registradas } \\
\text { em um centro de } \\
\text { assistência } \\
\text { toxicológica }\end{array}$ & $\begin{array}{l}\text { Caracterizar as internações } \\
\text { masculinas com diagnóstico de } \\
\text { intoxicação registradas em um } \\
\text { centro de informação e } \\
\text { assistência toxicológica do } \\
\text { Paraná. }\end{array}$ \\
\hline 3 & SILVA, 2014 & $\begin{array}{l}\text { Homens envenenados } \\
\text { como sujeitos do } \\
\text { cuidar e dos cuidados } \\
\text { de Enfermagem }\end{array}$ & $\begin{array}{l}\text { Caracterizar e analisar os } \\
\text { envenenamentos por carbamato } \\
\text { (chumbinho) em homens. }\end{array}$ \\
\hline 4 & $\begin{array}{l}\text { MEDEIROS; } \\
\text { MEDEIROS; } \\
\text { SILVA, } 2014\end{array}$ & $\begin{array}{l}\text { Intoxicação aguda por } \\
\text { agrotóxicos } \\
\text { anticolinesterásicos na } \\
\text { cidade do Recife, } \\
\text { Pernambuco, 2007- } \\
2010\end{array}$ & $\begin{array}{l}\text { Descrever aa características } \\
\text { epidemiológicas e a distribuição } \\
\text { espacial dos casos de } \\
\text { intoxicações agudas por } \\
\text { agrotóxicos anticolinesterásicos } \\
\text { na cidade do Recife, estado de } \\
\text { Pernambuco. }\end{array}$ \\
\hline 5 & PAVANATTO et & Contribuições & Conhecer as contribuições do \\
\hline
\end{tabular}




\begin{tabular}{|c|c|c|c|}
\hline & al., 2015 & $\begin{array}{l}\text { cuidado lúdico em } \\
\text { enfermagem na } \\
\text { desintoxicação } \\
\text { química devido ao uso } \\
\text { de crack }\end{array}$ & $\begin{array}{l}\text { cuidado lúdico em enfermagem } \\
\text { no estímulo à aceitação da } \\
\text { desintoxicação química pelo uso } \\
\text { do crack na percepção das } \\
\text { pessoas em processo de } \\
\text { desintoxicação. }\end{array}$ \\
\hline 6 & $\begin{array}{l}\text { DOMINGOS, et } \\
\text { al., } 2016\end{array}$ & $\begin{array}{ll}\text { Internações } & \text { por } \\
\text { intoxicação } & \text { de } \\
\text { crianças de zero a } 14 \\
\text { anos em hospital em } \\
\text { ensino no Sul do } \\
\text { Brasil, 2006 - } 2011\end{array}$ & 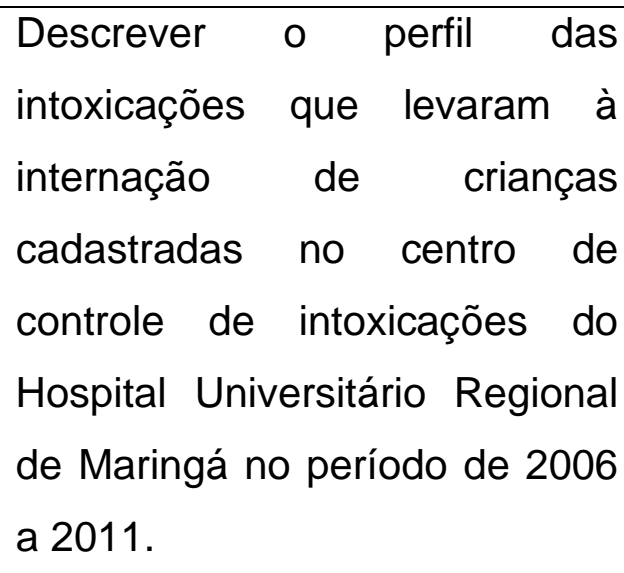 \\
\hline 7 & $\begin{array}{l}\text { SILVA; COELHO; } \\
\text { PINTO, } 2016\end{array}$ & $\begin{array}{l}\text { Fatores associados } \\
\text { aos óbitos entre } \\
\text { homens envenenados } \\
\text { por carbamato } \\
\text { (chumbinho) }\end{array}$ & $\begin{array}{l}\text { Determinar os fatores } \\
\text { associados aos óbitos em } \\
\text { vítimas de envenenamento por } \\
\text { carbamato (chumbinho). }\end{array}$ \\
\hline 8 & $\begin{array}{l}\text { VELOSO et al., } \\
2017\end{array}$ & $\begin{array}{l}\text { Violência autoinfligida } \\
\text { por intoxicação } \\
\text { exógena em um } \\
\text { serviço de urgência e } \\
\text { emergência }\end{array}$ & $\begin{array}{l}\text { Analisar as violências } \\
\text { autoinfligidas por intoxicação } \\
\text { exógena notificadas em um } \\
\text { serviço de saúde. }\end{array}$ \\
\hline 9 & $\begin{array}{l}\text { MAIOR; } \\
\text { OSORIO-DE- } \\
\text { CASTRO; } \\
\text { ANDRADE, } 2017\end{array}$ & $\begin{array}{l}\text { Internações por } \\
\text { intoxicações } \\
\text { medicamentosas em } \\
\text { crianças menores de } \\
\text { cinco anos no Brasil, } \\
2003 \text { - } 2012\end{array}$ & $\begin{array}{l}\text { Descrever as internações } \\
\text { hospitalares devidas às } \\
\text { intoxicações medicamentosas } \\
\text { em menores de cinco anos de } \\
\text { idade no Brasil, de } 2003 \text { a } 2012 \text {. }\end{array}$ \\
\hline 10 & $\begin{array}{l}\text { MURAKAMI } \\
\text { al., } 2017\end{array}$ & $\begin{array}{l}\text { Intoxicações crônica } \\
\text { por agrotóxico em } \\
\text { fumicultores }\end{array}$ & $\begin{array}{l}\text { Pesquisar intoxicações crônicas } \\
\text { por agrotóxicos e a relação com } \\
\text { o processo de trabalho na }\end{array}$ \\
\hline
\end{tabular}




\begin{tabular}{|c|c|c|c|}
\hline & & & fumicultura. \\
\hline 11 & $\begin{array}{l}\text { GERMANO; } \\
\text { ALONZO, } 2017\end{array}$ & $\begin{array}{l}\text { Estudo descritivo dos } \\
\text { atendimentos } \\
\text { hospitalares por } \\
\text { eventos toxicológicos } \\
\text { em um município do } \\
\text { estado de São Paulo, } \\
2012\end{array}$ & $\begin{array}{l}\text { Descrever } \\
\text { epidemiológico }\end{array}$ \\
\hline 12 & $\begin{array}{l}\text { TAVEIRA; } \\
\text { ALBUQUERQUE, } \\
2018\end{array}$ & $\begin{array}{l}\text { Análise das } \\
\text { notificações de } \\
\text { intoxicações agudas, } \\
\text { por agrotóxicos, } 38 \\
\text { municípios do estado } \\
\text { do Paraná }\end{array}$ & $\begin{array}{l}\text { Verificar a correlação entre } \\
\text { consumo de agrotóxicos em } \\
\text { municípios do Paraná e } \\
\text { notificação se intoxicações } \\
\text { agudas. }\end{array}$ \\
\hline
\end{tabular}

Fonte: Elaboração Própria

A amostra final foi identificada pelas seguintes revistas: Revista Gaúcha de Enfermagem, Escola Anna Nery Revista de Enfermagem, Epidemiologia Serviço Saúde e Saúde Debate.

O ano que teve o maior quantitativo de publicações foi 2017 apresentando quatro artigos publicados, seguido pelos anos de 2013, 2014, e 2016 onde obtevese dois artigos cada ano. Os anos de 2015 e 2018 contabilizaram apenas um artigo cada. O reconhecimento dos artigos mostrou que a base de dados SciELO predominou no número de publicações com 8 artigos. A LILACS e BDENF apresentaram apenas 2 artigos cada.

Dentre os artigos selecionados, em apenas um artigo aparece em seu título a palavra intoxicação medicamentosa, sendo que outro material apresenta o termo desintoxicação química, 8 artigos abordam em seus títulos palavras como Intoxicação exógena, Eventos Toxicológicos, Intoxicação Aguda e Intoxicação Crônica, 2 artigos aparecem em seus títulos o termo envenenamento. 


\section{DISCUSSÃO}

Primeiramente, é importante entender que as intoxicações exógenas são causadas por diversos agentes tóxicos que se apresentam clinicamente por diversos efeitos nocivos, sendo prejudicial aos diferentes sistemas devido a grande diversidade de substâncias.

O gênero masculino é apontado com uma maior vulnerabilidade para o envenenamento. Ressalta-se que as intoxicações estão entre os acidentes que causam muitas mortes entre crianças de um a 14 anos no Brasil (TAVARES et al.,2013).

Alguns estudos não relataram a via pela qual ocorreu a exposição as substâncias, porém outros materiais apresentam a via oral como a mais comum para a aquisição da substância tóxica (TAVARES et al., 2013; SILVA et al., 2014; VELOSO et al., 2017).

Nas intoxicações exógenas, dentre elas o uso de medicamentos e pesticidas apresentam-se como um problema de saúde pública em consequência de sua predominância. Homologando estes dados Veloso et al. (2017) revelam que pesquisas nacionais e internacionais apresentam as intoxicações exógenas como o principal meio utilizado nas investidas de suicídio, estando entre os três principais métodos mais escolhido nos casos de violência auto-infligida.

Diferentes são as situações que podem aumentar o risco para intoxicação exógena em ambos sexos, porém o desemprego, empobrecimento, escolaridade, abuso de substâncias, humor depressivo e problemas emocionais foram apontados por diversos autores na literatura como fatores predispostos para as ocorrências de envenenamento (SILVA et al., 2014).

Tais fatores ressaltam o quão é importante que a equipe de enfermagem deverá trabalhar a necessidade do resgate da autoestima e da busca de valores de cada paciente avaliado, implementando assim uma assistência de qualidade com uma visão holística a cada pessoa (PAVANATTO et al., 2015).

Salienta-se que as mulheres estão entre as que mais realizam tentativas de intoxicações exógenas em comparação aos homens, porém o sexo masculino predispõe a um resultado com mais frequência de mortalidade, seja este agravo pela maior facilidade de conseguir agentes tóxicos, pelo baixo nível de escolaridade ou por uma maior dificuldade de acessar os serviços de saúde (VELOSO et al., 2017). 
Dentre os produtos que possuem maior problema no Brasil estão os carbonatos que são usados de forma irregular no país, dentre estes problemas os mais identificados estão às intoxicações por envenenamento decorrente da ingestão de carbonatos como o chumbinho, tal intoxicação exógena por chumbinho é considerado um grande problema de mortalidade no Brasil por ser de fácil acesso, baixo custo, e grande efeito na tentativa de homicídio mesmo estando proibido a comercialização desta substância (SILVA; COELHO; PINTO, 2016).

Ressalta-se, que os medicamentos são considerados as substãncias tóxicas que mais causam intoxicação exógenaentre os principais agentes.

Corroborando com este resultado Domingos et al. (2016) traz que os medicamentos foram os principais agentes envolvidos nas intoxicações que levaram à internações.

Neste ínterim, as classes de medicamentos que mais destacaram nas ocasiões de intoxicações foram os fármacos não específicos, antiepilépticos, sedativos, hipnóticos e antiparkinsonianos, antibióticos sistêmicos e analgésicos/antitérmicos não opiáceis (MAIOR; CASTRO; ANDRADE, 2017).

Contrapondo-se a estes dados acima citado, a pesquisa de Germano e Alonzo (2017) realizada na cidade de Itapira-SP afirma que o principal agente tóxico envolvido nos casos de hospitalizações refere-se ao uso abusivo de drogas. A subnotificação dos eventos toxicológicos no referido estudo traz diferentes causas para a falta de informações como: a falta de qualidade no preenchimento dos registros, a escassez de informações, a falta de detalhes nas notificações e até mesmo pela falta de formação de profissionais da saúde qualificados para este tipo de problema.

Segundo Tavares e Albuquerque (2018) as intoxicações agudas são apontadas como os agravos de baixa procura assistencial por parte da população, ocorrendo em muitos casos falha no diagnóstico médico e uma subnotificação dos casos ocorridos. Em muitos dos casos de tentativa de suicídio por ingesta de agentes tóxicos a principal substância apontada por Medeiros, Medeiros e Silva (2014) para intoxicação aguda estão os agrotóxicos anticolinesterásicos.

Atualmente, é preocupante a utilização prolongada de diferentes agentes tóxicos pelo fato destes estarem relacionado a exposição exacerbada de agrotóxicos, considera-se que está exposição em muitos casos está relacionada ao aparecimento de agravos como: câncer, doenças neurológicas, respiratórias, 
alterações mutagênicas, teratogênicas e outras. Sendo as ocorrências de transtornos psiquiátricos menores como a perda auditiva neurosensorial e polineuropatia tardia induzida por organisfosforado as três manifestações mais relevantes nas intoxicações crônicas (MURAKAMI et al., 2017).

Vale salientar que as causas de envenenamento proporcionam um impacto social na população afetada, os custos com os serviços de saúde também devem ser consideradas, já que muitos dos casos necessita de hospitalizações. Sendo necessário compreender melhor o tipo de ocorrência por parte da equipe de enfermagem, tornando de suma importância um planejamento de ação educativa eficiente com orientações de medidas que visam a restrição e o acesso a agentes tóxicos para prevenção de intoxicações (REIS et al., 2013).

Diante do abordado é de grande importância que o enfermeiro e a equipe de saúde estejam aptos a buscar informações relevantes ao caso do paciente e tomar medida de intervenções adequadas para cada caso, tomando-se como ferramenta o histórico de enfermagem importante na coleta de dados, como também tomar medidas de proteção durante a prestação da assistência junto ao cliente. Assim, para prestar uma assistência de qualidade os profissionais de enferamgem podem realizar diversos cuidados de urgência como: manter vias áreas pérvias, aspirar secreção oral, separa materiais de emergência, auxiliar em preocedimentos de alta complexidade dependendo do caso da vítima (PAVANATTO et al., 2015).

\section{Conclusão}

O primeiro atendimento prestado a vítimas de intoxicação exógena é crucial, sendo determinante para o bom andamento do quadro clínico da vítima, de forma que a partir da eficácia da conduta inicial pode evoluir positivamente ou negativamente, a depender do preparo da equipe e condições da vítima.

Considera-se o papel da enfermagem como primordial no contexto do atendimento multiprofissional ao paciente com diagnóstico de intoxicação exógenas de suas diversas causas.

É importante realizar estudos voltados para assistência de enfermagem em pacientes com intoxicação exógena, visto que os mesmos ainda se encontram escassos, para melhor contribuir no preparo e qualificação dos profissionais de enfermagem na abordagem e assistência à vítima desse agravo. 
Esse estudo foi pertinente pela apresentação das publicações brasileiras que evidenciaram sobre os cuidados de enfermagem na intoxicação exógena.

\section{Referências}

BRASIL, Ministério da Saúde. Guia de Vigilância Epidemiológica. Brasília- Secretaria de Vigilância Epidemiológica, 2014.

DOMINGOS, Samara Messias et al. Internações por intoxicação de crianças de zero a 14 anos em hospital de ensino no sul do Brasil, 2006-2011. Epidemiol. Serv. Saúde, v. 25, n. 2, p. 343-350, Abr-Jun., 2016. Disponível em: < http://www.scielo.br/pdf/ress/v25n2/22379622-ress-25-02-00343.pdf> Acesso em: 04 Jan. 2019.

GERMANO, Lucas Coraça; ALONZO, Herling Gregorio Aguilar. Estudo descritivo dos atendimentos hospitalares por eventos toxicológicos em um município do estado de São Paulo, 2012. Epidemiol. Serv. Saúde, v. 26, n. 3, p. 545-556, Jul-Set., 2017. Disponível em: < http://www.scielo.br/pdf/ress/v26n3/2237-9622-ress-26-03-00545.pdf> Acesso em: 14 Jan. 2019.

MAIOR, Maria da Cunha Lobo Souto; OSORIO-DE-CASTRO, Claudia Garcia Serpa; ANDRADE, Carla Lourenço Tavares de. Internações por intoxicações medicamentosas em crianças menores de cinco anos no Brasil, 2003-2012. Epidemiol Serv Saúde, v. 26, n. 4, p. 771-782, 2017. Disponível em: <http://scielo.iec.gov.br/pdf/ess/v26n4/2237-9622-ess-2604-00771.pdf > Acesso em: 14 Jan. 2019.

MEDEIROS, Márcia Noelle Cavalcante; MEDEIROS, Marília Cavalcante; SILVA, Maria Beatriz Araújo. Intoxicação aguda por agrotóxicos anticolinesterásicos na cidade do Recife, Pernambuco, 2007-2010. Epidemiol. Serv. Saúde, v. 23, n. 3, p. 509-518, Jul-Set., 2014.Disponível em: <http://www.scielo.br/pdf/ress/v23n3/1679-4974-ress-23-03-00509.pdf> Acesso em: 14 Jan. 2019.

MELO, Wyana Ferreira et al. Assistência de Enfermagem à vítima de intoxicação exógena. Revista Brasileira de educação e saúde, v. 5, n. 2, p. 26 - 31, Abr-Jun., 2015.Disponível em: < https://www.gvaa.com.br/revista/index.php/REBES/article/view/4020/4180 > Acesso em: 04 Jan. 2019. 
MURAKAMI, Yumie et al. Intoxicação crônica por agrotóxicos em fumicultores. Saúde Debate, v. 41, n. 113, p. 563-576, Abr-Jun., 2017.Disponível em: < http://www.scielo.br/pdf/sdeb/v41n113/0103-1104-sdeb-41-113-0563.pdf > Acesso em: 04 Jan. 2019.

PAVANATTO, Paola Aparecida et al. Contribuições do cuidado lúdico em enfermagem na desintoxicação química devido ao uso de crack. Rev Gaúcha Enferm, v. 36, n. 2, p. 50-5,

Jun., $2015 . \quad$ Disponível em: < https://seer.ufrgs.br/RevistaGauchadeEnfermagem/article/view/48736 > Acesso em: 14 Jan. 2019.

REIS, Lúcia Margarete dos et al. Saúde do homem: Internações hospitalares por intoxicação registradas em um centro de assistência toxicológica. Esc Anna Nery, v. 17, n. 3, p. 505511, Jul-Set., 2013.Disponível em: < http://www.scielo.br/pdf/ean/v17n3/1414-8145-ean-1703-0505.pdf > Acesso em: 04 Jan. 2019.

SANTOS, Renato Reis; PEREIRA NETO, Omar; CUNHA, Cristiane Martins. Perfil de vítimas de intoxicações exógenas agudas e assistência de enfermagem. Revista de Enfermagem Atenção à Saúde, v. 4, n. 2, p. 45-55, Ago/Dez., 2015. Disponível em: <http://seer.uftm.edu.br/revistaeletronica/index.php/enfer/article/view/978/pdf > Acesso em: 04 Jan. 2019.

SANTOS, Simone Agadir; LEGAY, Letícia Fortes; LOVISI, Giovanni Marcos. Substâncias tóxicas e tentativas e suicídios: considerações sobre acesso e medidas restritivas. Cad. Saúde Colet, v. 21, n. 1, p. 53-61, 2013. Disponível em: < http://www.scielo.br/pdf/cadsc/v21n1/a09.pdf > Acesso em: 04 Jan. 2019.

SILVA, Júlio César Santos de et al. Homens envenenados como sujeitos do cuidar e dos cuidados de enfermagem. Escola Anna Nery Revista de Enfermagem, v. 18, n. 4, OutDez., 2014. Disponível em: <http://www.scielo.br/pdf/ean/v18n4/1414-8145-ean-18-040716.pdf > Acesso em: 04 Jan. 2019.

SILVA, Júlio César; COELHO, Maria José; PINTO, Cecília Maria Izidoro. Fatores associados aos óbitos entre homens envenenados por carbamato (chumbinho). Rev Gaúcha Enferm, v. $37, \quad$ n. 2 , p. e54799, Jun., 2016. Disponível em: < http://www.scielo.br/pdf/rgenf/v37n2/0102-6933-rgenf-1983-144720160254799.pdf> Acesso em: 04 Jan. 2019. 
SOARES, Cassia Baldini et al. Revisão integrativa: conceitos e métodos utilizados na enfermagem. Rev Esc Enferm USP, v. 48, n. 2, p. 335-45., 2014. Disponível em: < http://www.scielo.br/pdf/reeusp/v48n2/pt_0080-6234-reeusp-48-02-335.pdf > Acesso em: 14 Jan. 2019.

SOUZA, Marcela Tavares de; SILVA, Michelly Dias da; CARVALHO, Rachel. Revisão integrativa: o que é e como fazer. Einstein, v. 8, n. 1, p. 102-6., 2010. Disponível em: < http://www.scielo.br/pdf/eins/v8n1/pt_1679-4508-eins-8-1-0102.pdf > Acesso em: 14 Jan. 2019.

TAVARES, Érika Okuda et al. Fatores associados à intoxicação infantil. Escola Anna Nery, v. 17, n. 1, p. 31-37, Jan-Mar., 2013. Disponível em: < http://www.scielo.br/pdf/ean/v17n1/05.pdf > Acesso em: 14 Jan. 2019.

TAVARES, Erika Okuda; OLIVEIRA, Magda Lúcia Félix de. Padrões mínimos de atendimento inicial à urgência toxicológica para abordagem à criança intoxicada. Rev Rene, v.13, n. $1, \quad$ p. 147-57, 2012. Disponível em: < http://www.periodicos.ufc.br/rene/article/view/3785 > Acesso em: 04 Jan. 2019.

TAVEIRA, Bruna Letícia Souza; ALBUQUERQUE, Guilherme Souza Cavalcanti de. Análise das notificações de intoxicações agudas, por agrotóxicos, em 38 municípios do estado do Paraná. Saúde Debate, v. 42, n. 4, p. 211-222, Dez., 2018. Disponível em: < http://www.scielo.br/pdf/sdeb/v42nspe4/0103-1104-sdeb-42-spe04-0211.pdf > Acesso em: 04 Jan. 2019.

VELOSO, Caique et al. Violência autoinfligida por intoxicação exógena em um serviço de urgência e emergência. Ver Gaúcha Enferm, v. 38, n. 2, p. e66178, 2017. Disponível em: < http://www.scielo.br/pdf/rgenf/v38n2/0102-6933-rgenf-1983-144720170266187.pdf > Acesso em: 04 Jan. 2019. 